\title{
Estudio morfológico del páncreas de alpacas (Vicugna pacos) jóvenes y adultas
}

\author{
MORPHOLOGIC STUDY OF THE PANCREAS OF YOUNG AND ADULTS ALPACAS \\ (Vicugna pacos)
}

\author{
Gianmarco Panesi A. ${ }^{1}$, Alexander Chávez R. ${ }^{1,3}$, Miluska Navarrete Z. ${ }^{1}$, Nieves \\ Sandoval C. ${ }^{2}$, Alberto Sato S. ${ }^{1}$, Francisco Santos R. ${ }^{1}$
}

\section{Resumen}

\begin{abstract}
El objetivo de este estudio fue realizar una caracterización morfológica del páncreas de alpacas jóvenes $(n=9)$ y adultas $(n=5)$. Para el estudio macroscópico se observó el páncreas in situ reconociendo su forma y relación con otros órganos. Para el estudio microscópico se realizaron cortes histológicos de $5 \mu \mathrm{m}$ de espesor tiñendo las láminas con hematoxilina de Mayer-Eosina y tricrómico de Masson. El páncreas se divide en tres secciones: cuerpo, lóbulo derecho y lóbulo izquierdo. El lóbulo izquierdo se extiende hacia el plano izquierdo llegando a colindar con el bazo y en mayor proporción al colon descendente. El conducto hepatopancreático presentó dos orígenes: uno donde el conducto hepático se introduce en el parénquima pancreático y lo abandona por el lóbulo derecho como conducto hepatopancreático, y otro donde el conducto pancreático se une con el hepático al emerger del lóbulo derecho para formar el conducto hepatopancreático. En la microscopía se apreció que el parénquima pancreático se encuentra rodeado por una fina capa de tejido conectivo formada en su mayoría por fibras colágenas, que se invaginan dentro del parénquima formando lóbulos y pseudolóbulos, así como células con forma piramidal acomodadas en forma de acinos. Dentro del parénquima se observaron los islotes de Langerhans distribuidos al azar. Los conductos intralobulares e interlobulares se encuentran conformados por epitelio cubico simple, y el conducto hepatopancreático está conformado por una extensa mucosa irregular tapizada de epitelio columnar con microvellosidades. Se concluye que el páncreas de la alpaca muestra diferencias morfológicas con otras especies como rumiantes, herbívoros y carnívoros domésticos.
\end{abstract}

Palabras clave: alpaca; morfología; páncreas

\footnotetext{
${ }^{1}$ Laboratorio de Anatomía Animal y Fauna Silvestre, ${ }^{2}$ Laboratorio de Histología, Embriología y Patología Veterinaria, Facultad de Medicina Veterinaria, Universidad Nacional Mayor de San Marcos, Lima, Perú

${ }^{3}$ E mail: alexander.chavez@unmsm.edu.pe
}

Recibido: 28 marzo de 2017

Aceptado para publicación: 5 de octubre de 2017 
The aim of this study was to morphologically characterize the pancreas of young $(\mathrm{n}=9)$ and adult $(\mathrm{n}=5)$ alpacas. In the macroscopic study, the pancreas was observed in situ to determine the shape and its relationship with other organs. In the microscopic study, routine histologic procedures were applied and histological sections of $5 \mu \mathrm{m}$ thick were stained with Mayer-Eosin hematoxylin and Masson trichrome. The pancreas is divided in three sections: body, right lobe and left lobe which extend to the left plane, bordering the spleen and in greater proportion to the descendant colon. The hepatopancreatic duct presented two origins; one where the hepatic duct enters the pancreatic parenchyma and it leaves by the right lobe as the hepatopancreatic duct and another where the pancreatic duct joins with the hepatic duct as it emerges from the right lobe to form the hepatopancreatic duct. In the microscopic evaluation was observed that the pancreatic parenchyma is surrounded by a thin layer of connective tissue formed mostly by collagen fibers, which invaginate within the parenchyma forming lobes and pseudolobules, as well as cells with pyramidal shape accommodated in the form of acini. In the parenchyma was observed the islets of Langerhans randomly distributed. The intralobular and interlobular ducts were formed by simple cubic epithelium, and the hepatopancreatic duct was formed by an extensive irregular mucosa covered with columnar epithelium with microvilli. It is concluded that the alpaca pancreas shows morphological differences with other species such as ruminants, herbivores and domestic carnivores.

Key words: alpaca; morphology; pancreas

\section{INTRODUCCIÓN}

Las alpacas al igual que otros camélidos sudamericanos (CSA) habitan las zonas altoandinas. Cerca del total de la población de alpacas se encuentra en la sierra peruana (INEI, 2012). Las alpacas, y los CSA en general, son el principal medio de sustento para muchos productores de escasos recursos en los países andinos de Sudamérica, incluyendo Ecuador, Perú, Bolivia, Argentina y Chile (Quispe et al., 2009, 2011), toda vez que estos animales convierten con eficiencia la vegetación nativa en carne y fibra de alta calidad (Iñiguez et al., 1998).

Los CSA son los primeros mamíferos de larga vida con valores altos de glucosa que no desarrollan las complicaciones vistas en la diabetes humana (Cebra et al., 2006). No obstante, y a pesar de la importancia socioeconómica de la alpaca, no existen trabajos referentes a las características morfológicas de órganos vitales como el páncreas.
El presente estudio morfológico busca aportar una visión macroscópica y microscópica del páncreas de la alpaca para su posterior aplicación en disciplinas afines como fisiología, patología, nutrición y cirugía.

\section{Materiales y Métodos}

Se emplearon cadáveres de 14 alpacas de descarte, entre jóvenes y adultas de raza Huacaya, de la estación experimental IVITAMaranganí, ubicada en la provincia de Canchis, departamento de Cusco, Perú. Para el estudio se formó un grupo de jóvenes conformado por nueve machos de 16 a 18 meses de edad, y un grupo de adultos conformado por dos machos y tres hembras con edades entre 24 y 63 meses.

La eutanasia y disección de los animales se realizó en la sala de necropsia de la estación experimental respetando las normas de bienestar animal y con la autorización del 
Comité de Ética de la Facultad de Medicina Veterinaria (FMV) de la Universidad Nacional Mayor de San Marcos (UNMSM) (Código 2017-002). Para el efecto, se administró acepromazina en dosis de $0.02 \mathrm{mg} / \mathrm{kg}$, vía endovenosa, para la sedación y pentobarbital sódico en sobredosis $(60 \mathrm{mg} / \mathrm{kg})$ como eutanásico.

En los cadáveres se procedió a la apertura de la cavidad abdominal mediante una incisión desde el xifoides hasta la región púbica, luego otra incisión bordeando los arcos costales con el fin de exponer las vísceras y determinar la ubicación in situ del páncreas. Se registraron las características externas del órgano y su relación con órganos colindantes y vasos que lo irrigan. En el páncreas, una vez extraído, se determinó su forma y se registró el peso mediante una balanza analítica Precix-Weight OVIT-III con una precisión de $10 \mathrm{mg}$, así como la longitud y diámetro de cada lóbulo y del cuerpo. Asimismo, la ubicación, longitud, diámetro y desembocadura del conducto hepatopancreático. Las medidas se tomaron con ayuda de un vernier digital.

Para el estudio histológico se realizaron tres cortes por cada lóbulo pancreático (extremo, medio y base), dos cortes por cuerpo y uno del conducto hepatopancreático, que fueron conservados en formol bufferado al $10 \%$. Las muestras fueron sometidas a procesos rutinarios para la evaluación histológica en el laboratorio de Histología de la FMVUNMSM. Se hicieron cortes de $5 \mu \mathrm{m}$ de espesor y las láminas fueron teñidas con hematoxilina de Mayer-Eosina y tricrómico de Masson. La lectura de las láminas se hizo con un microscopio binocular Leica.

\section{Resultados}

\section{Evaluación Macroscópica}

El páncreas se encuentra ubicado a nivel de la región del epigastrio dorsal, envuel- to y fijado a los órganos que lo rodean por el mesoduodeno, omento mayor, ligamento gastroesplénico y mesocolon. Es de color rosado pálido, de bordes irregulares y de apariencia lobular. Su consistencia es blanda. $\mathrm{La}$ forma del páncreas asemeja a una «Y» invertida que va de craneal a caudal. Presenta un cuerpo, lóbulo derecho e izquierdo. El cuerpo se encuentra situado a nivel del píloro del cual nacen los lóbulos derecho e izquierdo.

El lóbulo izquierdo está relacionado al compartimiento proximal del estómago, vena gastroesplénica, al hilio del bazo y, en mayor proporción, al colon descendente (Figura 1).

El lóbulo derecho está relacionado al hígado y duodeno. De este lóbulo emerge el conducto pancreático que recibe al conducto biliar, denominándose conducto hepatopancreático, el cual va por el borde mesentérico del duodeno proximal y desemboca en la ampolla duodenal. Entre este lóbulo y el hígado se puede apreciar un par de nódulos linfoides.

El origen del conducto hepatopancreático es variable, ya que se puede dar por el ingreso del conducto hepático al parénquima del lóbulo derecho, recibiendo dentro de este a los conductillos pancreáticos para salir por este mismo bajo el nombre de conducto hepatopancreático (Figura 2). Asimismo, se puede originar por la unión del conducto pancreático y el conducto hepático a nivel del vértice del lóbulo derecho, con una longitud aproximada de $2.4 \mathrm{~cm}$. y un diámetro aproximado de $0.62 \mathrm{~cm}$; desembocando en la ampolla duodenal que se ubica aproximadamente a $23 \mathrm{~cm}$ del píloro.

La irrigación del páncreas está dada por la arteria pancreático duodenal craneal, la cual irriga al cuerpo y lóbulo derecho. Además, por la arteria pancreático duodenal caudal, que irriga al lóbulo izquierdo, junto con ramas provenientes de la arteria esplénica. La inervación del páncreas está a cargo de las fibras autónomas que nacen del plexo iliaco. 


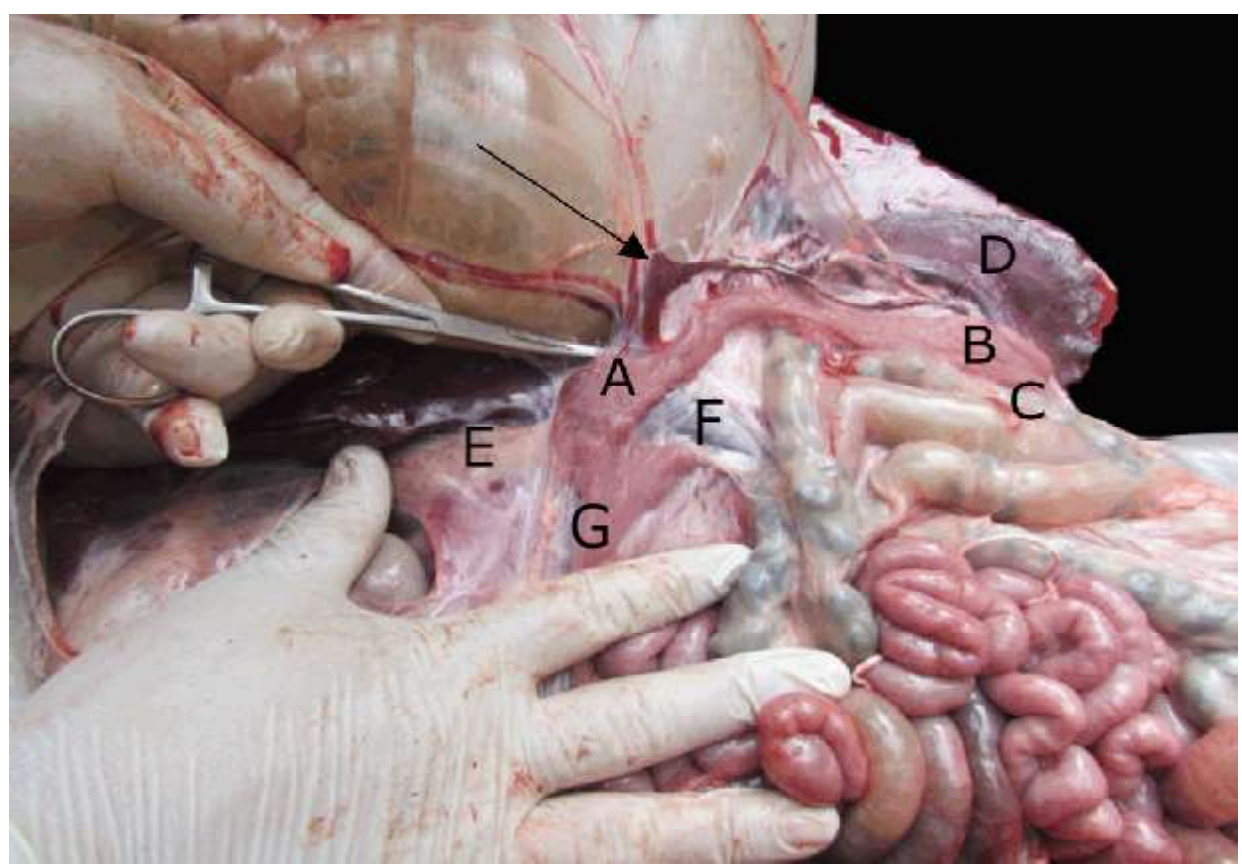

Figura 1. Vista macroscópica del páncreas de la alpaca. Ubicación de la base del lóbulo izquierdo (A) y vértice del lóbulo izquierdo (B), colindando con el colon descendente (C) y el bazo (D). También se puede apreciar el nódulo linfático hepatopancreático (E), la vena porta $(\mathrm{F})$ y el lóbulo derecho del páncreas $(\mathrm{G})$. La flecha indica la vena gastroesplénica

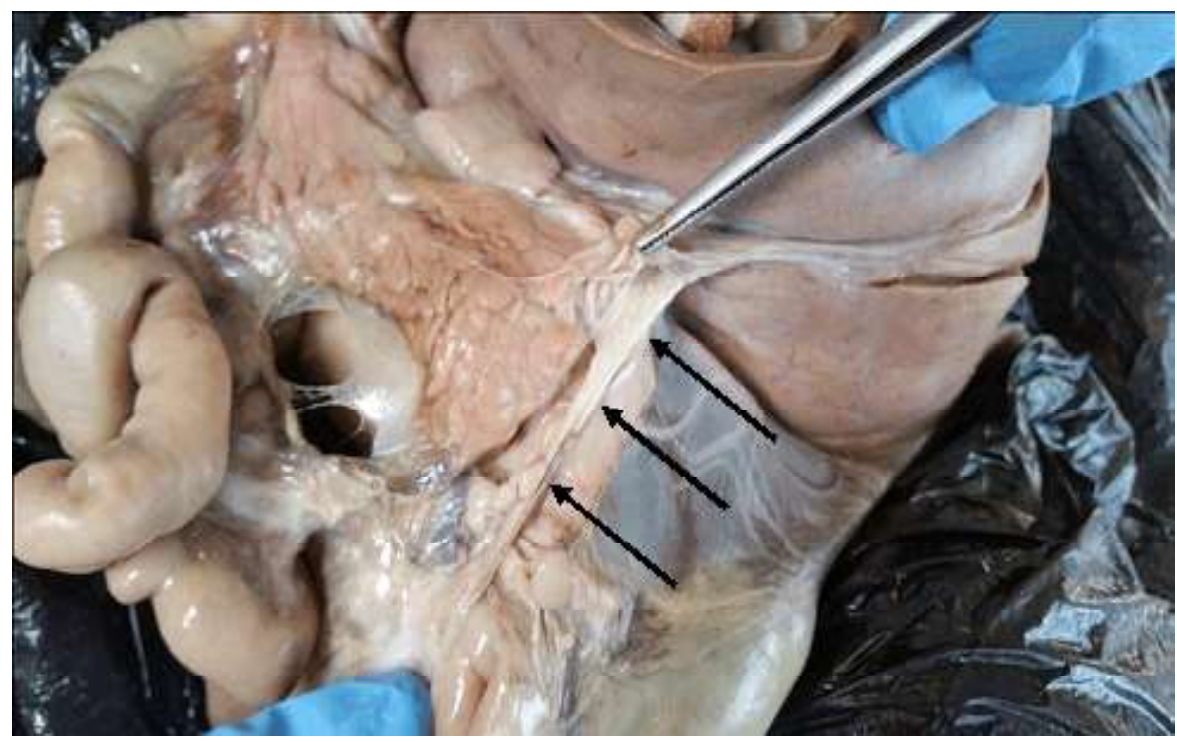

Figura 2. Vista del conducto hepatopancreático. Al debridar el lóbulo derecho se aprecia el conducto hepático recibiendo conductillos hepáticos para convertirse en el conducto hepatopancreático 


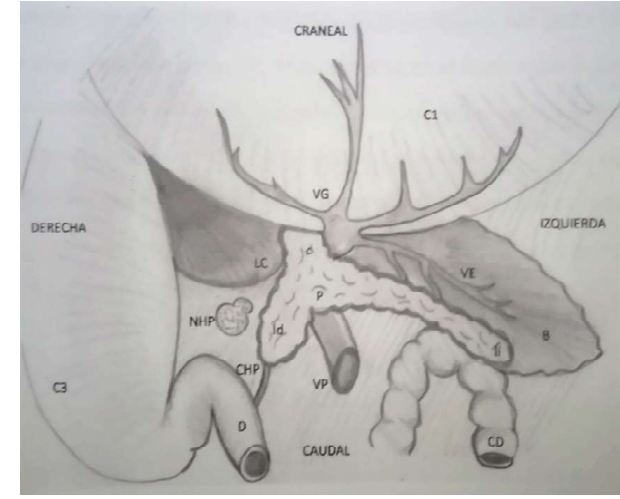

Figura 3. Representación gráfica de la topografía del páncreas. $\mathrm{C} 1$ : primer compartimento del estómago; VG: vena gástrica; $\mathrm{P}$ : páncreas; LC: lóbulo caudado del hígado; VE: vena esplénica; $\mathrm{B}$ : bazo; $\mathrm{CD}$ : colon descendente; VP: vena porta; CHP: conducto hepatopancreático; $\mathrm{D}$ : duodeno; $\mathrm{C} 3$ : tercer compartimento del estómago; NHP: nódulo hepatopancreático; c: cuerpo; ld: lóbulo derecho; li: lóbulo izquierdo (Fuente: Los autores)

En la Figura 3 se aprecia un resumen de la topografía del páncreas de la alpaca.

El lóbulo izquierdo es el más desarrollado de los lóbulos. En los jóvenes presenta entre 14 y $24 \mathrm{~cm}$ de longitud, mientras que el lóbulo derecho tiene una longitud entre 8 y 14 $\mathrm{cm}$ y el cuerpo entre 2.5 y $11 \mathrm{~cm}$. En los adultos, el lóbulo izquierdo presenta entre 15.4 y $25 \mathrm{~cm}$, el lóbulo derecho entre 6.9 y $12 \mathrm{~cm}$ y el cuerpo entre 3 y $10 \mathrm{~cm}$.

El peso aproximado del páncreas fue de 40 g. El diámetro varía según la porción de cada lóbulo. Para el estudio se tomaron las mediciones del extremo, cuerpo y vértice de los lóbulos izquierdo y derecho y una medición a la mitad del cuerpo pancreático (Cuadro 1). En el caso del grupo de adultos, se observó que el páncreas de las hembras era más grande que el de los machos.

\section{Evaluación Microscópica}

El páncreas se encuentra rodeado por un fino tejido conectivo, el cual se invagina dando la formación de lóbulos y pseudolóbulos.
Con la tinción de tricrómico de Masson se puede apreciar las fibras de colágeno (fibras basófilas) formando pseudolóbulos (Figura 4I). Este tejido conectivo también sirve para sostener vasos sanguíneos y conductos interlobulares; además, relaciona al páncreas con el nódulo linfático hepatopancreático.

\section{Páncreas exocrino}

El páncreas se considera una glándula mixta. La parte exocrina se clasifica como glándula túbulo-acinar. Las células que sintetizan las enzimas digestivas están ordenadas como racimos llamados ácinos glandulares. En los cortes histológicos normales, los ácinos glandulares son cortados de manera oblicua, lo cual hace difícil definir su forma. Las células del ácino glandular se observan en forma de cuña, y en coloración H\&E están conformadas por un núcleo redondo central teñido de azul (basófilo) y un citoplasma teñido de color rosa (acidófilo). En el ápice de las células se observan granulaciones teñidas de manera más intensa (Figura 4-II).

\section{Conducto pancreático}

Los conductos pancreáticos se pueden clasificar en cuatro tipos:

- Conductos intercalados: Reciben la secreción directamente del ácino glandular, y tienen un epitelio cúbico aplanado que se extiende hasta dentro del lumen del ácino glandular; las cuales se conocen como células centro-acinares (Figura 4-III).

- Conductos intralobulares: Tienen un epitelio cubico simple y se encuentran dentro de los lóbulos (Figura 4-II). Reciben las secreciones de los conductos intercalados.

- Conductos interlobulares: Se encuentran entre los lóbulos dentro del tejido conectivo y pueden variar considerablemente de tamaño. Las formas más pequeñas pueden tener epitelio cúbico y las formas más grandes presentan epitelio columnar. Estos recogen las secreciones desde los conductos intralobulares (Figura 4-III). 
Cuadro 1. Medidas y peso del páncreas de alpacas ${ }^{1}$

\begin{tabular}{|c|c|c|c|c|c|c|c|c|c|c|c|c|c|c|}
\hline \multirow[t]{2}{*}{ Sexo } & \multirow{2}{*}{$\begin{array}{c}\text { Peso } \\
(\mathrm{g})\end{array}$} & \multicolumn{3}{|c|}{$\begin{array}{c}\mathrm{LP} \\
(\mathrm{cm})\end{array}$} & \multicolumn{3}{|c|}{$\begin{array}{l}\text { CHP } \\
(\mathrm{cm})\end{array}$} & \multicolumn{7}{|c|}{$\begin{array}{c}\mathrm{DP} \\
(\mathrm{cm})\end{array}$} \\
\hline & & $\mathrm{LD}$ & LI & $\mathrm{C}$ & $\mathrm{L}$ & $\mathrm{D}$ & $\mathrm{X}$ & Cuerpo & LIE & LIM & LIB & LDE & LDM & LDB \\
\hline \multicolumn{15}{|c|}{ Jóvenes } \\
\hline M & 41.0 & 9 & 24 & 3 & 3 & 0.5 & 30 & 3.76 & 2.5 & 1.2 & 1.3 & 2.1 & 2 & 2.4 \\
\hline M & 37.1 & 10 & 20 & 10 & 1.5 & 0.7 & 21 & 1.26 & 1.6 & 0.7 & 1.3 & 1.9 & 1.1 & 1.7 \\
\hline $\mathrm{M}$ & 43.0 & 14 & 14 & 2.5 & 2 & 1.86 & 27 & 1.89 & 2 & 1.6 & 0.8 & 2.1 & 1.3 & 1.3 \\
\hline M & 30.0 & 12 & 19 & 4 & 3.3 & 1 & 20 & 1.63 & 1.9 & 1.5 & 1 & 2 & 1.4 & 1.8 \\
\hline $\mathrm{M}$ & 35.5 & 9 & 18 & 11 & 2 & 0.36 & 21 & 1.55 & 2.2 & 2 & 1.4 & 0.5 & 1.3 & 1.9 \\
\hline M & 39.4 & 8 & 19 & 6 & 2 & 0.42 & 19 & 2.2 & 2.2 & 1.9 & 1.3 & 1.7 & 1.2 & 1.2 \\
\hline M & 43.4 & 9 & 17 & 4 & 2 & 0.37 & 20 & 1.9 & 1.9 & 1.3 & 1.9 & 1.8 & 1.8 & 2.1 \\
\hline $\mathrm{M}$ & 28.3 & 9 & 20 & 10 & 1.5 & 0.49 & 20 & 1.8 & 1.8 & 1 & 1.9 & 1 & 1.5 & 2 \\
\hline M & 27.8 & 10 & 18 & 5 & 1.5 & 0.22 & 27 & 2 & 2 & 2 & 2 & 1.6 & 1.7 & 2 \\
\hline \multicolumn{15}{|c|}{ Adultos } \\
\hline M & 26.0 & 12 & 16 & 3 & 2 & 0.25 & 25 & 2.2 & 1.3 & 1.3 & 0.8 & 1.9 & 0.6 & 0.8 \\
\hline $\mathrm{M}$ & 43.1 & 12 & 22 & 5 & 3.5 & 0.3 & 21 & 2.4 & 1 & 1 & 1.3 & 2 & 1 & 1.2 \\
\hline $\mathrm{H}$ & 58.9 & 6.9 & 15.4 & 5 & 2.5 & 0.27 & 26 & 2.3 & 1.5 & 1.6 & 1.6 & 1.8 & 1.3 & 1.8 \\
\hline $\mathrm{H}$ & 54.4 & 6 & 24 & 10 & 4 & 1 & 23 & 2.78 & 1.7 & 1.9 & 1.9 & 1.7 & 0.9 & 2.6 \\
\hline $\mathrm{H}$ & 56.0 & 8 & 25 & 10 & 3 & 0.9 & 25 & 2.2 & 2.4 & 2.1 & 2.1 & 2.3 & 1.6 & 2.4 \\
\hline
\end{tabular}

${ }^{1}$ M: Macho, H: Hembra, LP: longitud del páncreas, CHP: conducto hepatopancreático, DP: diámetro del páncreas, LD: lóbulo derecho, LI: lóbulo izquierdo, C: cuerpo, L: longitud, D: diámetro, X: distancia del píloro a la desembocadura del conducto hepatopancreático, LIE: lóbulo izquierdo extremo, LIM: lóbulo izquierdo medio, LIB: lóbulo izquierdo base, LDE: lóbulo derecho extremo, LDM: lóbulo derecho medio, LDB: lóbulo derecho base

- Conductos hepatopancreáticos: Presentan una mucosa formada por epitelio cilíndrico simple con microvellosidades. En la submucosa se puede apreciar abundante cantidad de ácinos glandulares rodeados de tejido muscular liso (Figura 5-I).

\section{Páncreas endocrino}

Los islotes de Langerhans se apreciaron en un corte con H\&E y con objetivo de 10X. Se observaron como zonas teñidas acidófilas más pálidas entremezcladas con los acinos glandulares. Las células endocrinas dentro de los islotes son células poliédricas de núcleo central basófilo y citoplasma acidófilo claro, las cuales no presentan granulaciones en su interior. Además, se encuentran distribuidas como cordones irregulares alrededor de capilares, los cuales reciben las hormonas secretadas. Con un objetivo de 40x se puede apreciar una rica vascularización del islote, evidenciado por racimos de células sanguíneas y células endoteliales (Figura 5-II).

\section{Discusión}

El color del páncreas de la alpaca in vivo es rosa pálido, lo cual difiere de las descripciones realizadas por Navarrete y Sato 


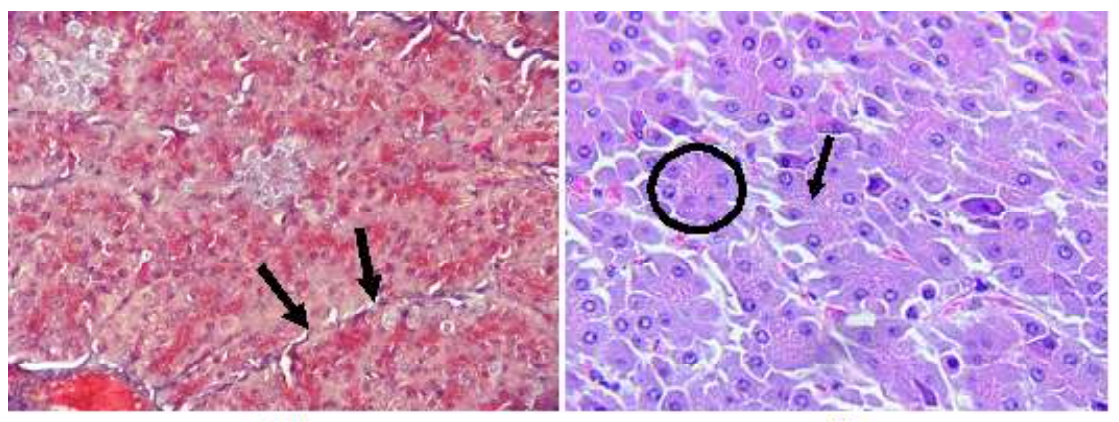

(I)

II

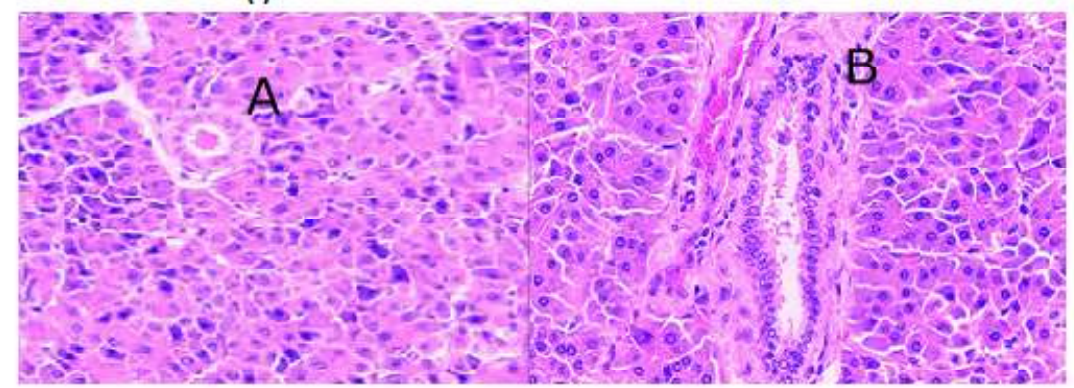

III

Figura 4. Páncreas de alpaca. (I) Las flechas muestran el tejido conectivo formando pseudolóbulos (tricrómico de Masson, 400X). (II) El círculo encierra un ácino glandular y la flecha muestra el núcleo de una célula centroacinar (H\&E, 400X). (III) A: Conducto intralobular. B: Conducto interlobular (H\&E, 400X)

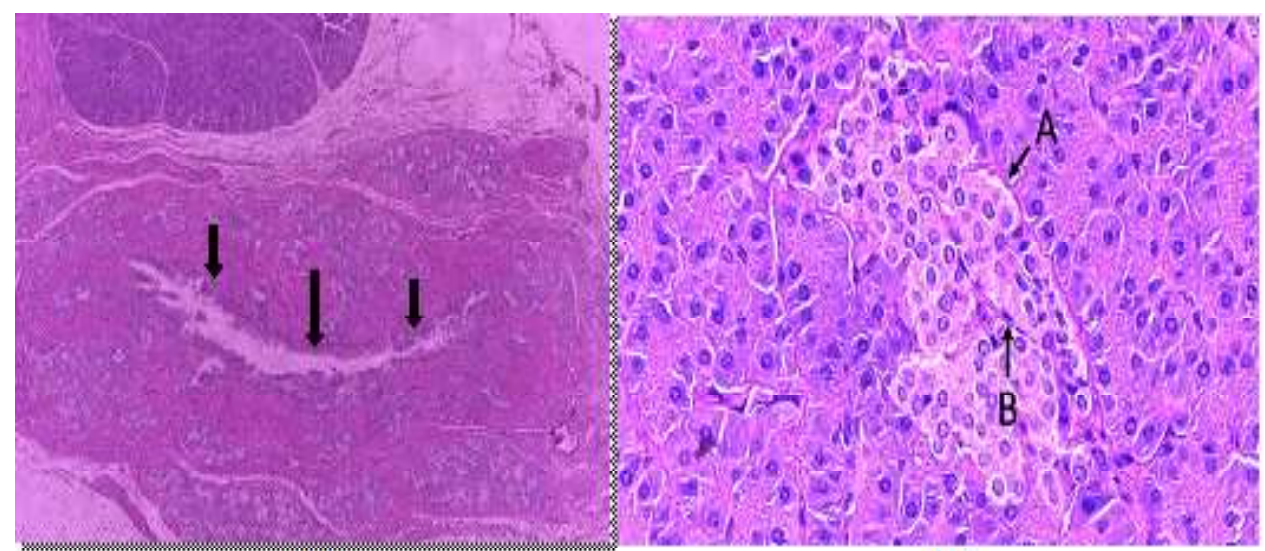

(I)

(III)

Figura 5. Páncreas de la alpaca. (I) Conducto hepatopancreático (H\&E. 100x). (II) Islote de Langerhans rodeado de células sanguíneas (A) y rodeando células endoteliales (B) (H\&E, 400X)

(2010); debido posiblemente a que ese trabajo fue realizado en órganos que pasaron por un proceso de conservación.

A diferencia de los carnívoros, los cuales tienen un páncreas en forma de «V» (König y Liebich, 2004) ya que prácticamente presentan dos lóbulos, el páncreas de la alpaca tiene forma de «Y» invertida, el cual rodea a la vena porta. Esto se contrasta con la descripción del páncreas del camello (Ali y Masaad, 2007) de forma cuadrilátera rodeando la vena porta con una extensión glandular en forma de puente que va desde el lóbulo derecho al lóbulo izquierdo. 
En los carnívoros, rumiantes, cerdos y equinos, el páncreas se encuentra en la línea media con la totalidad o gran mayoría de la masa pancreática hacia el lado derecho. En el caso de la alpaca, el lóbulo izquierdo que representa la mayor cantidad de la masa pancreática se extiende hacia el lado izquierdo, llegando a colindar con el bazo y el colon descendente, siendo similar al del camello.

El origen del conducto hepatopancreático se puede dar como lo descrito por Ghezzi et al. (2000) en llamas, pero difiere de lo descrito en camellos por Ali y Masaad (2007), quienes indican que el conducto pancreático nace del cuerpo del páncreas para unirse inmediatamente al conducto hepático para formar el conducto hepatopancreático. No obstante, también se puede dar por el ingreso del conducto hepático, a través del lóbulo derecho, para emerger por este mismo bajo el nombre de conducto hepatopancreático.

El páncreas de alpaca se encuentra rodeado de una fina capa de tejido conectivo formado en su mayoría por fibras colágenas, las cuales se invaginan en el parénquima formando lóbulos y pseudolóbulos, a diferencia del camello que presenta una cápsula formada por una gruesa capa de tejido conectivo con abundante tejido adiposo (Ali y Masaad, 2007).

La distribución de los ácinos glandulares es similar a las descritas en otras especies de rumiantes, herbívoros y carnívoros domésticos. En forma similar, los islotes de Langerhans se presentaron de diferentes formas, tamaños y distribuidos al azar en el parénquima, sin que se observasen islotes en zonas interlobulares, como se ha descrito en los bovinos (Bonner y Like, 1980; Cebra et al., 2006).

El conducto hepatopancreático muestra una mucosa irregular con abundantes glándulas mucosas y está tapizado por un epitelio cilíndrico con microvellosidades, tal y como lo describen Ali y Masaad (2007) en came- llos y Ghezzi et al. (2000) en llamas. Las microvellosidades observadas en el epitelio del conducto hepatopancreático no han sido descritas anteriormente.

\section{Conclusiones}

- El páncreas de las alpacas es diferente al de otras especies domésticas, incluyendo los camélidos del viejo mundo, en cuanto a su forma, cuerpo prominente y gran tamaño del lóbulo izquierdo.

- No se apreció diferencias en el tamaño del páncreas entre alpacas jóvenes y adultas.

- Las características del conducto hepatopancreático es similar al descrito en camellos y llama; sin embargo, el origen puede variar según el individuo.

- Microscópicamente, el páncreas de la alpaca es similar al páncreas de otras especies de rumiantes, herbívoros y carnívoros domésticos.

- Microscópicamente, el conducto hepatopancreático es diferente al conducto de otras especies de rumiantes, herbívoros y carnívoros domésticos, siendo similar al del camello y llama.

\section{Agradecimiento}

Los autores agradecen al FONDECYT CONCYTEC por el financiamiento del estudio a través del Convenio 097-2014.

\section{Literatura Citada}

1. Ali G, Masaad GAM. 2007. A comparative morphological study on the pancreas of the dromedary and the donkey. MSc Thesis. Sudan: University of Khartoum. $160 \mathrm{p}$.

2. Bonner-Weir S, Like AA. 1980. A dual population of islets of Langerhans in bovine pancreas. Cell Tissue Res 206: 157-170. doi: 10.1007/BF00233616 
3. Cebra CK, Bildfell RJ, Fischer KA. 2006. Microanatomic features of pancreatic islets and immunolocalization of glucose transporters in tissues of 1lamas and alpacas. Am J Vet Res 67: 524528. doi: 10.2460/ajvr.67.3.524

4. Ghezzi MD, Alzola RH, Lupidio MC, Massone A, Castro AN, Rodríguez JA. 2000. Conducto hepatopancreático de la llama (Lama glama). Rev Chil Anat 18(1): 27-34. doi: 10.4067/S071698682000000100004

5. [INEI] Instituto Nacional de Estadística e informática. 2012. Resultados definitivos. IV Censo Nacional Agropecuario 2012. [Internet]. Disponible en: http://proyectos.inei.gob.pe/web/ D o c u m e n o s P u b i cos/ ResultadosFinalesIVCENAGRO.pdf

6. Iñiguez L, Alem R, Wauer A, Mueller J. 1998. Fleece types, fiber characteristics and production system of an outstanding llama population from
Southern Bolivia. Small Ruminant Res 30: 57-65. doi: 10.1016/S09214488(98)00079-0

7. Konig HE, Liebich H. 2004. Veterinary anatomy of domestic mammals: textbook and colour atlas. Germany: Schattauer. 787 p.

8. Navarrete M, Sato A. 2010. Aspectos anatómicos de la cría de alpaca. En: Sanidad de alpacas en la etapa neonatal. Manual para estudiantes y profesionales de veterinaria. España: Univ Complutense. p 51-62.

9. Quispe E. 2011. Adaptaciones hematológicas de los camélidos sudamericanos que viven en zonas de elevadas altitudes. Rev Complutense Cienc Vet 5(1). 1-26.

10. Quispe EC, Rodríguez TC, Iñiguez LR, Mueller JP. 2009. Producción de fibra de alpaca, llama, vicuña y guanaco en Sudamérica. Anim Genet Resour Inform 45: 1-14. 